\title{
Measurement of spin diffusion in semi-insulating GaAs
}

\author{
C. P. Weber, 1 a) Craig A. Benko, ${ }^{1, b)}$ and Stanley C. Hiew ${ }^{1}$ \\ Department of Physics, Santa Clara University, 500 El Camino Real, Santa Clara, \\ CA 95053-0315
}

(Dated: 22 April 2022)

We use optical transient-grating spectroscopy to measure spin diffusion of optically oriented electrons in bulk, semi-insulating GaAs(100). Trapping and recombination do not quickly deplete the photoexcited population. The spin diffusion coefficient of $88 \pm 12 \mathrm{~cm}^{2} / \mathrm{s}$ is roughly constant at temperatures from $15 \mathrm{~K}$ to $150 \mathrm{~K}$, and the spin diffusion length is at least $450 \mathrm{~nm}$. We show that it is possible to use spin diffusion to estimate the electron diffusion coefficient. Due to electron-electron interactions, the electron diffusion is 1.4 times larger than the spin diffusion.

The following article appeared in Journal of Applied Physics and may be found at http: //link.aip.org/link/?jap/109/106101

Copyright 2011 American Institute of Physics. This article may be downloaded for personal use only. Any other use requires prior permission of the author and the American Institute of Physics.

Keywords: Spin diffusion; Semi-insulating GaAs; Transient grating

The burgeoning field of semiconductor spintronics relies on moving spin-polarized electrons through distances comparable to the dimensions of an electronic device. The importance of spin transport has led to several studies of spin diffusion in GaAs quantum wells. Spin transport in quantum wells can differ markedly from the bulk material, due to to different scattering rates and especially to the different spin-orbit coupling 1 ? Nonetheless, there have been relatively few measurements 4$]$ of spin diffusion in bulk GaAs. In $n$-doped samples with $n=1 \times 10^{16}$ and $2 \times 10^{16} \mathrm{~cm}^{-3}$, the spin diffusion coefficient $D_{s}$ ranged from 10 to $200 \mathrm{~cm}^{2} / \mathrm{s}$.

Spin diffusion in semi-insulating GaAs (SI-GaAs) has not been reported. SI-GaAs has been proposed as a platform for nuclear spintronics ${ }^{5}$ due to its low carrier density. Moreover, Kikkawa et al. showed that electrons could be optically oriented in SI-GaAs and would subsequently diffuse into an adjacent ZnSe film, maintaining their spin polarization ${ }^{6}$. Since SI-GaAs is a ubiquitous substrate material for thin film growth and for spintronic devices, such spin diffusion is of practical consequence, whether intentional or not. In this work, we find that SI-GaAs has a large, temperature-independent spin diffusion coefficient.

We measured spin diffusion with an ultrafast transient spin grating 7 , which measures the decay rate $\gamma_{s}$ of a spin-density wave (the "grating") with wavelength $\Lambda$ and wavevector $q=2 \pi / \Lambda$. The grating amplitude decays - through spin relaxation, electron-hole recombination, and diffusion - at a rate of

$$
\gamma_{s}(q)=D_{s} q^{2}+1 / \tau_{0}
$$

\footnotetext{
a) Electronic mail: cweber@scu.edu

b) Present address: JILA, National Institute of Standards and Technology, and University of Colorado, Department of Physics, 440 UCB, Boulder, CO 80309, USA
}

Here, $D_{s}$ is the spin diffusion coefficient, and $\tau_{0}$ is the lifetime for trapping, recombination, and spin relaxation. Measurement at several $q$ determines $D_{s}$. We measure in a reflection geometry, and improve the detection efficiency by heterodyne detection ${ }^{8}$. Noise is further suppressed by $95 \mathrm{~Hz}$ modulation of the grating phase and lock-in detection 9 .

The SI-GaAs sample was grown by Wafer Technology. It was undoped, oriented (100), had room temperature resistivity $\rho \geq 10^{7} \Omega$-cm and Hall mobility $\mu_{H} \geq 5000$ $\mathrm{cm}^{2} / \mathrm{V}$-s.

The pump and probe pulses came from a mode-locked Ti:Sapphire laser with wavelength near $800 \mathrm{~nm}$ and repetition rate of $80 \mathrm{MHz}$. The two pump pulses were focused to a spot of $65 \mu \mathrm{m}$ diameter with total fluence 3.0 $\mu \mathrm{J} / \mathrm{cm}^{2}$ except as indicated; probe pulses were always a factor of 2.5 weaker. Assuming one photoexcited electron per absorbed photon in a $1 \mu \mathrm{m}$ absorption length 10 , we photoexcite $\sim 8.5 \times 10^{16} \mathrm{~cm}^{-3}$ carriers, greater than the typical concentration of deep traps in SI-GaAs 11 . In this way we are able to measure motion of free carriers at times longer than the trapping time, and to use the density of photoexcited electrons as an estimate of the free-carrier density.

Figure 1 shows typical results of spin-grating measurements. After dropping rapidly for $0.5 \mathrm{ps}$, the diffracted signal - and the spin grating amplitude - decays exponentially at rate $\gamma_{s}$. Higher- $q$ gratings decay more quickly, as expected for diffusive motion. The solid lines show fits of the data to the form $A+B \exp \left[-\gamma_{s}(q) t\right]$. The size of the constant offset $A$ averages $2.5 \%$ of the exponential decay (and never exceeds $7 \%$ ), so it does not significantly influence the values of $\gamma_{s}$. We speculate that the offset may arise from a small fraction of localized carriers.

We determined spin diffusion coefficients by fitting $\gamma_{s}(q)$ to Eq. 11, as shown in Fig. 27. The measured 


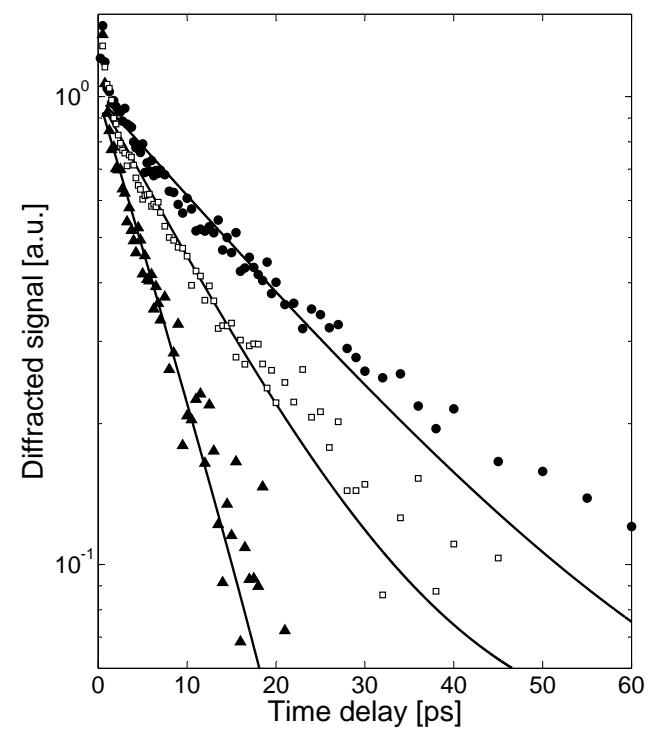

FIG. 1. Decay of the transient spin grating at $15 \mathrm{~K}$ (semilog scale). Curves correspond to wavevectors $q=2.01 \times 10^{4}, 3.14$ $\times 10^{4}$, and $4.53 \times 10^{4} \mathrm{~cm}^{-1}$ (slowest to fastest). Solid lines are a least-squares fit to an exponential decay plus a constant offset.

$D_{s}$ represents electron spin diffusion with no appreciable contribution from the holes. The near-bangap absorption of circularly polarized light by GaAs excites spinpolarized electrons and holes, but the hole spins rapidly randomize, leaving behind spin-aligned electrons ${ }^{12}$. By fitting to times after the rapid initial decay, we obtain $\gamma_{s}$ for electrons only. Moreover, the photoexcited electron and hole populations are spatially uniform, so the electrons' motion is not hindered by electron-hole Coulomb attraction, and spin diffusion may occur more quickly than ambipolar diffusion ${ }^{7}$. Uniform excitation also precludes any photorefractive grating.

The $15 \mathrm{~K}$ data in Figs. 1 and 2 a were all taken with pump fluence of $3.0 \mu \mathrm{J} / \mathrm{cm}^{2}$. The signal size decreases at higher temperatures, so data were taken with fluences of up to $9.0 \mu \mathrm{J} / \mathrm{cm}^{2}$. We found that fluence had a small effect on grating decay rate: as compared to $3.0 \mu \mathrm{J} / \mathrm{cm}^{2}$, decay rates measured at 6.0 to $9.0 \mu \mathrm{J} / \mathrm{cm}^{2}$ were typically slower by $5-20 \%$, while attenuating to $1.5 \mu \mathrm{J} / \mathrm{cm}^{2}$ did not change $\gamma_{s}$ at all. The origin of this fluence-dependent grating decay is a topic of further research. However, the measured changes in $\gamma_{s}$ are sufficiently small that they do not add much uncertainty to the measured $D_{s}$.

Fig. $2 \mathrm{p}$ shows the values of $D_{s}$ at several temperatures as determined from the fits to Eq. 1. The spin diffusion is roughly constant in temperature, and it is fast - comparable to that previously seen ${ }^{9}$ at low temperature in a quantum well with $\mu=69000 \mathrm{~cm}^{2} / \mathrm{V}$-s. This supports our conclusion that most photoexcited electrons remain mobile for times of at least $\tau_{0}$. (The values of $\tau_{0}$ were $60,100,28$, and 23 ps at $15,80,110$, and 150 $\mathrm{K}$, respectively.) Noting that the spin lifetime $\tau_{s} \geq \tau_{0}$, the spin diffusion length $L_{s}=\sqrt{D_{s} \tau_{s}}$ is at least $450 \mathrm{~nm}$.
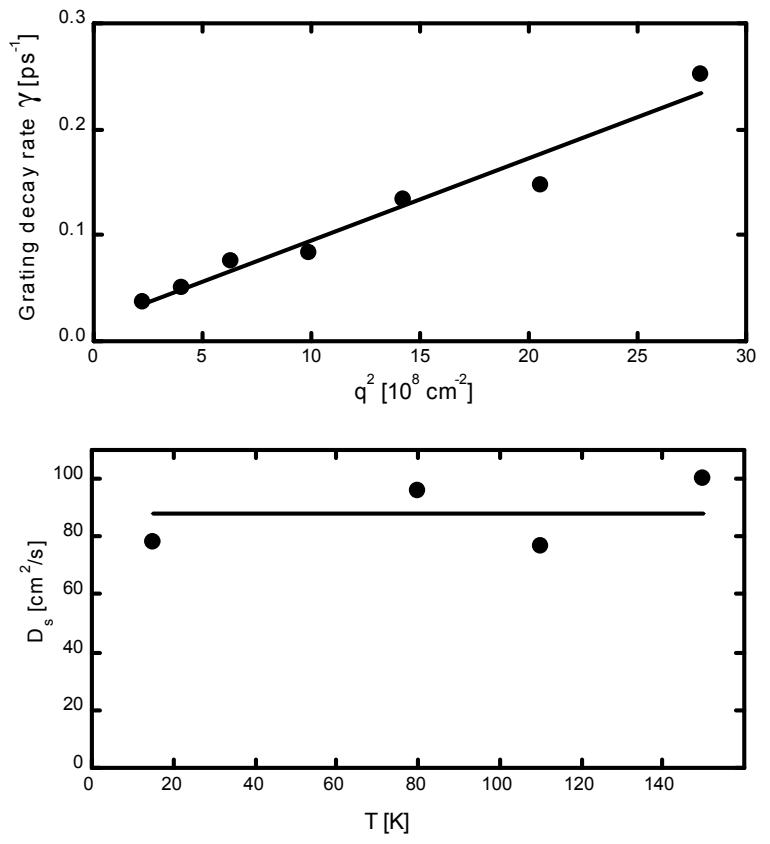

FIG. 2. (a): Decay rate of the spin grating vs $q^{2}$ at $15 \mathrm{~K}$. The line is a least-squares fit to the form of Eq. 1, indicating diffusive behavior with $D_{s}=78 \mathrm{~cm}^{2} / \mathrm{s}$. (b): Spin diffusion coefficient vs temperature. The line is the mean value of 88 $\mathrm{cm}^{2} / \mathrm{s}$.

This length, comparable to the optical absorption length, suggests the possibility of efficient spin injection from SIGaAs into thin epilayers ${ }^{6}$.

Finally, we infer electron diffusion from the measured spin diffusion. One must consider spin Coulomb drag ${ }^{\sqrt{13}}$, the effect of electron-electron collisions that transfer momentum between counter-diffusing spin-up and spindown populations. This effect has been observed to suppress spin diffusion, relative to electron diffusion, by factors up to 8 in high-mobility quantum wells ${ }^{9}$. The electron diffusion is 14 :

$$
D_{e}=\frac{D_{s}}{\chi_{0} / \chi_{s}-\chi_{0} e^{2} \rho_{\uparrow \downarrow} D_{s}} .
$$

Here $\chi_{s}$ is the spin susceptibility, $\chi_{0}=\partial n / \partial \mu$ is the electronic susceptibility, and $\rho_{\uparrow \downarrow}$ is the spin transresistivity. Our inferred value of $D_{e}$ thus should be regarded as an estimate, since it depends - through $\chi_{s}, \chi_{0}$ and $\rho_{\uparrow \downarrow}$ - on the density $n$ and heating $\Delta T$ of the photoexcited electrons, which are known only approximately 15 .

We estimate $\rho_{\uparrow \downarrow}(T)$ from Fig. 1 of Ref. 14 and calculate $\chi_{0}(T)$ numerically ${ }^{16}$ for a noninteracting electron gas of density $n=8.5 \times 10^{16} \mathrm{~cm}^{-3}$ assuming spherical, parabolic bands. We use the Perdew-Wang parametrization ${ }^{177}$ for the exchange-correlation energy to obtain $\chi_{0} / \chi_{s} \approx 0.82$. Eq. 2 gives values of $D_{e}$ ranging from 110 to $140 \mathrm{~cm}^{2} / \mathrm{s}$. $D_{e}$ is consistently about $40 \%$ higher than $D_{s}$, showing the importance of electronelectron interactions even in this high-resistivity material. 
As a check on $D_{e}$, we convert diffusion to mobility using the Einstein relation, $\mu_{e}=e \chi_{0} D_{e} / n$. We find that $\mu_{e}$ increases from $7500 \mathrm{~cm}^{2} / \mathrm{V}$-s at the highest measured temperature to $13000 \mathrm{~cm}^{2} / \mathrm{V}$-s at the lowest. We are not aware of any measurement of electron mobility in SI-GaAs at low temperature. However, our mobility agrees reasonably with that of undoped n-GaAs at low temperature $\frac{18}{18}$, while the room-temperature mobilities of SI-GaAs and of undoped n-GaAs are comparable ${ }^{11 / 18 \mid 19}$. This rough agreement shows that transient spin gratings can complement transport in measuring $D_{e}$. The technique might be particularly useful in ferromagnets with a large anomalous Hall effect.

Our observation of roughly temperature-independent spin diffusion contrasts with the apparent strong temperature dependence in $n$-doped GaAs: a sample with $n=2 \times 10^{16} \mathrm{~cm}^{-3}$ had $D_{s}=10 \mathrm{~cm}^{2} / \mathrm{s}$ at $4 \mathrm{~K}$ (Ref. 3), while one with $n=1 \times 10^{16} \mathrm{~cm}^{-3}$ had $D_{s}=200$ $\mathrm{cm}^{2} / \mathrm{s}$ at room temperature ${ }^{4}$. In the absence of electronelectron interactions, the spin diffusion would equal the charge diffusion, $n \mu / e \chi_{0}$. Lowering temperature decreases $n / \chi_{0}$, but generally increases the mobility, two effects that partly cancel. At low $T, n / \chi_{0}$ approaches a nonzero minimum value due to degeneracy. Since our photoexcited density exceeds the densities studied in $n$ doped samples, degeneracy sets in at a higher temperature. Thus for the same $\mu(T)$ we would expect diffusion to in our samples to equal that of $n$-doped samples at 150 $\mathrm{K}$, while exceeding it by a factor of 4 at low temperature. Differing mobility among our semi-insluating sample and the two $n$-doped samples reported likely accounts for the remaining differences in $D_{s}$.

Semi-insulating GaAs is an important substrate for spintronic materials and structures, and may also be useful for nuclear spintronics. We have measured its electron spin diffusion coefficient, $D_{s}$, under conditions in which most photoexcited carriers are not trapped. This coefficient, which is difficult to learn from transport measurements, measures the motion of electron spins. $D_{s}$ has a high value of $\sim 88 \mathrm{~cm}^{2} / \mathrm{s}$, independent of temperature.

Our result suggests that SI-GaAs could be useful for injecting spin into adjacent layers. It also holds a lesson for optical-orientation experiments in which a film is measured on top of a SI-GaAs substrate: unless a barrier-layer is grown, spins in the substrate will likely diffuse into the material being measured. Finally, we have shown that one can use spin diffusion to estimate electron diffusion, if one takes proper account of electron degeneracy (through $\chi_{0}$ ), and of electron-electron interactions through the spin susceptibility and spin Coulomb drag.

\section{ACKNOWLEDGMENTS}

The authors thank G. Vignale for illuminating conversations and for sending a program to evaluate $\chi_{0} / \chi_{s}$. We thank Joe Orenstein for loaned equipment, R. A. Kaindl for a loaned Ti:Sapphire laser, and R. P. Campion for sending the sample. This research was supported by an award from Research Corporation.

${ }^{1}$ C. P. Weber, J. Orenstein, B. A. Bernevig, S. C. Zhang, J. Stephens, and D. D. Awschalom, Physical Review Letters 98, 4 (2007)

${ }^{2}$ J. M. Kikkawa and D. D. Awschalom, Nature 397, 139 (1999)

${ }^{3}$ S. A. Crooker, M. Furis, X. Lou, C. Adelmann, D. L. Smith, C. J. Palmstrom, and P. A. Crowell, Science 309, 2191 (2005)

${ }^{4}$ H.-L. Yu, X.-M. Zhang, P.-F. Wang, H.-Q. Ni, Z.-C. Niu, and T. Lai, Applied Physics Letters 94, 202109 (2009)

${ }^{5}$ J. A. Reimer, Solid State Nuclear Magnetic Resonance 37, 3 (2010)

${ }^{6}$ J. M. Kikkawa, J. A. Gupta, I. Malajovich, and D. D. Awschalom, Physica E: Low-Dimensional Systems \& Nanostructures 9, 194 (2001)

${ }^{7}$ A. R. Cameron, P. Riblet, and A. Miller, Physical Review Letters 76, 4793 (1996)

${ }^{8}$ P. Vohringer and N. F. Scherer, Journal of Physical Chemistry 99, 2684 (1995)

${ }^{9}$ C. P. Weber, N. Gedik, J. E. Moore, J. Orenstein, J. Stephens, and D. D. Awschalom, Nature 437, 1330 (2005)

${ }^{10}$ M. D. Sturge, Physical Review 127, 768 (1962)

${ }^{11}$ G. M. Martin, J. P. Farges, G. Jacob, J. P. Hallais, and G. Poiblaud, Journal of Applied Physics 51, 2840 (1980)

${ }^{12} \mathrm{~F}$. Meier and B. Zakharchenya, Optical Orientation (NorthHolland, Amsterdam, 1984)

${ }^{13}$ I. D'Amico and G. Vignale, Europhysics Letters 55, 566 (2001)

${ }^{14}$ I. D'Amico and G. Vignale, Physical Review B-Condensed Matter 65, 085109/1 (2002)

${ }^{15} \mathrm{In}$ what follows we assume electronic heating of $\Delta T=30 \mathrm{~K}$. We have checked that the value of $D_{e}$ depends only weakly $(8 \%)$ on $\Delta T$ in the range $0 \mathrm{~K} \leq \Delta T \leq 200 \mathrm{~K}$. Varying $n$ from $8.5 \times 10^{15}$ to $1.2 \times 10^{17} \mathrm{~cm}^{-3}$ changes $D_{e}$ by no more than $7 \%$. The inferred $\mu_{e}$, on the other hand, varies strongly with $n$ and roughly as $1 /(T+\Delta T)$.

${ }^{16}$ N. Mohankumar and A. Natarajan, Physica Status Solidi B-Basic Research 188, 635 (1995)

17 J. P. Perdew and Y. Wang, Physical Review B 45, 13244 (1992)

${ }^{18}$ Rode, D. L., in Semiconductors and Semimetals, Vol. 10: Transport Phenomena, edited by R. K. Willardson and A. C. Beer (Academic Press, 1975).

${ }^{19}$ C. H. Gooch, C. Hilsum, and B. R. Holeman, Journal of Applied Physics 32, 2069 (1961) 